\section{Cahiers de littérature orale}

$83 \mid 2018$

Geneviève Calame-Griaule

\title{
La littérature orale dans des articulations pluridisciplinaires et multi-aréales
}

Acknowledging of Oral Literature as a Full-Fledged Scientific Domain and Discipline, Pluriareal Approach

\section{Ursula Baumgardt}

\section{(2) OpenEdition}

\section{Journals}

Édition électronique

URL : https://journals.openedition.org/clo/4701

DOI : $10.4000 /$ clo.4701

ISSN : 2266-1816

Éditeur

INALCO

Édition imprimée

Date de publication : 21 juin 2018

Pagination : 35-54

ISBN : 9782858313143

ISSN : 0396-891X

\section{Référence électronique}

Ursula Baumgardt, "La littérature orale dans des articulations pluridisciplinaires et multi-aréales »,

Cahiers de littérature orale [En ligne], 83 | 2018, mis en ligne le 20 juin 2019, consulté le 30 juin 2021.

URL : http://journals.openedition.org/clo/4701 ; DOI : https://doi.org/10.4000/clo.4701

\section{(c) (7) (5)}

Cahiers de littérature orale est mis à disposition selon les termes de la Licence Creative Commons Attribution - Pas d'Utilisation Commerciale 4.0 International. 


\title{
La littérature orale dans des articulations pluridisciplinaires et multi-aréales
}

\author{
Ursula BAUMGARDT \\ PLIDAM, Inalco, Sorbonne Paris Cité
}

Geneviève Calame-Griaule s'est toujours intéressée à la conception de la parole dans différentes sociétés. Elle prête aussi une attention particulière aux productions textuelles des sociétés de l'oralité. Dans son célèbre article « Pour une étude ethnolinguistique de la littérature orale » (1970), elle pose les bases d'une approche pluridisciplinaire de la littérature orale et mentionne la coexistence de celle-ci avec la littérature écrite en langues africaines, notamment celle qui utilise la graphie arabe.

En restant prioritairement dans le domaine africaniste, je voudrais donner un bref aperçu de quelques travaux réalisés en littérature orale dans la continuité de cette approche qui demeure une référence incontournable pour l'étude de l'oralité. Cet aperçu qui ne se veut pas exhaustif ${ }^{1}$ montrera par la diversité des champs d'application concernés jusqu'à quel point l'approche de G. Calame-Griaule est féconde. Il illustrera la nécessité de poursuivre le travail bibliographique précieux et systématique commencé par Veronika Görög-Karady en 1981 et 1992, mais qui n'a pas été continué dans cette perspective. Je rendrai en même temps compte de certaines interrogations nouvelles et de quelques difficultés concernant notamment la reconnaissance de la littérature orale comme relevant d'une discipline scientifique. Par la même occasion, je voudrais suggérer quelques perspectives pour les recherches en littérature orale.

1. Je ne peux pas aborder ici les travaux portant spécifiquement sur la performance en littérature orale, et notamment ceux en ethnomusicologie. 


\section{Travaux dans la continuité de l'approche de Geneviève Calame-Griaule}

L'aire culturelle de référence privilégiée des premières études est l'Afrique de l'Ouest, étant donné que les membres de son équipe, l'URA 1024, sont surtout spécialistes de ces aires culturelles, comme Roger Labatut, Christiane Seydou, Veronika Görög-Karady, Suzy Platiel, Jean Derive, Diana Rey-Hulman, mais également Paulette Roulon-Doko².

\section{Le conte}

Les travaux probablement les plus nombreux concernent le conte, par exemple Suzy Platiel (1984) La Fille volage et autres contes du pays san, ou Suzanne Ruelland (1973) La Fille sans mains, les Contes d'origine et autres contes gbaya (1977) de Paulette Roulon ou bien les publications sur les contes igbo de Françoise Ugochukwu (1992 et 2006). Ils se situent dans la même perspective que les Contes dogon du Mali bien connus de son équipe, dont la publication en édition bilingue a été réalisée en 2006, bien après la constitution de cette précieuse documentation.

Outre la collecte et l'édition de corpus - qui garde toujours son importance ${ }^{3}$ - l'approche thématique et comparative est privilégiée, comme dans son ouvrage Des cauris au marché (1987) qui réunit plusieurs de ses articles. C'est le cas également du travail collectif ${ }^{4}$ en son hommage, Graines de parole (1989), ou bien de L'Enfant, du Mariage, et de L'Univers familial dans le conte africain, édités par Veronika Görög-Karady (respectivement 1988, 1994, 1997), ou encore de La mort dans les contes seereer d'Amade Faye (1997). L'approche thématique est approfondie par les réflexions sur l'importance structurale d'un élément donné, comme c'est le cas dans les Histoires d'Enfants Terribles (1980). Ces recherches ouvrent à leur tour la voie à l'approfondissement de l'approche théorique du conte-type à travers l'exemple de La Fille difficile 5 .

2. Paulette Roulon-Doko travaille essentiellement sur la Centrafrique.

3. Je voudrais signaler ici le souci de poursuivre et d'élargir la documentation sur les contes de différentes régions de l'Afrique, concrétisé à travers la collection « Contes et légendes » (en français), créée en 1980 par Patrick Mérand aux éditions Karthala et dirigée par Henry Tourneux depuis 1985-1986.

4. L'ouvrage Paroles nomades. Ecrits d'ethnolinguistique africaine, DerIve \& BAUMGARDT, 2006, s'inscrit également dans cette perspective.

5. GÖRÖG-KaRADY \& SEYdou, 2000. 
La collecte du répertoire d'une seule énonciatrice, en l'occurrence celui d'une conteuse, représentant une unité textuelle cohérente et relativement étendue ${ }^{6}$ permet d'établir entre autres le cadre référentiel dans lequel s'inscrivent les textes, et de formuler des hypothèses plus précises sur la façon dont l'énonciateur s'approprie le patrimoine oral, y opère un choix et se profile comme créateur exprimant une vision du monde personnelle.

Dans d'autres domaines culturels, on peut signaler le travail d'Eva Toulouze (2003) sur l'art verbal des populations de la Sibérie, ou celui de Vincent Durand-Dastès qui s'intéresse à une vaste collecte de contes en Chine. Il analyse la portée idéologique de cette collecte organisée par le parti communiste chinois, l'objectif de l'organisation politique étant de s'appuyer sur le patrimoine oral pour faire passer les nouveaux contenus promus par le parti ${ }^{7}$.

\section{L'épopée}

Un genre souvent étudié est l'épopée. Des travaux concernant ce genre dans une perspective ethnolinguistique connaissent déjà une longue tradition, comme en témoignent de nombreuses recherches : Christiane Seydou (1972, 1976, 2010a et b), Lilyan Kesteloot (et al.) (1971, 1972), Gérard Dumestre (1979), Jan Jansen (2001), Bassirou Dieng (1993) ainsi que l'ouvrage transversal dirigé par Lilyan Kesteloot et Bassirou Dieng, Les Épopées d'Afrique Noire (1997). Les chercheurs continuent à s'y intéresser. On peut citer par exemple Jean Derive (2002), Sandra Bornand (2005), Alpha Ousmane Barry (2011) et Alpha Oumarou Ba (2011). De même, la discussion sur la classification générique épique se poursuit, comme c'est le cas du travail de Oumar Ndiaye (2010) sur le pekaan, poésie des pêcheurs du fleuve Sénégal.

L'épopée fait également l'objet de recherches actuelles, comme en témoigne la thèse en cours d'Amadou Sow, « Le héros épique peul dans la chanson traditionnelle et moderne poulâr ». Le genre bénéficie d'un vif intérêt, favorisé entre autres par la création du Réseau Euro-Africain de Recherche sur l'Épopée (REARE) en 2000 à Dakar, à l'initiative de Lilyan Kesteloot ${ }^{8}$.

\section{BAUMGARDT, 2000.}

\section{Durand-Dastès, 2014.}

8. Le REARE a organisé le colloque international « La temporalité dans les épopées : structuration, fonctions et modes d'expression », Inalco, Paris, 22-23 septembre 2016, quelques jours avant le colloque en hommage à Geneviève Calame-Griaule. 


\section{Les Genres brefs}

Les textes moins souvent analysés sont ceux qu'on classe généralement dans la rubrique des genres brefs et qui, pourtant, présentent un intérêt considérable. En témoignent, par exemple, les travaux de Cécile Leguy sur les proverbes bwa du Mali (2001), qu'elle aborde toujours par rapport à la situation dans laquelle ils sont énoncés. J.-C. Nkejahabizi propose l'analyse des wellérismes dans la littérature en kinyarwanda (2013). On peut citer également Abdoulaye Keïta et son analyse de la poésie d'exhortation en wolof au Sénégal par les lutteurs avant, voire après le combat (2010a et b). Un tout autre domaine est étudié par Jean Derive (2014), la poésie amoureuse en dioula (Côte d'Ivoire), en situant les chants de manière précise tels qu'ils sont produits tout au long des différentes étapes que comprend le mariage. Catherine Servan-Schreiber de son côté étudie la tradition de plaisanteries dans les chants bhojpuri (Inde du Nord) (1981) et s'intéresse aussi bien aux chanteurs itinérants dans cette aire culturelle (1999) qu'à la façon d'introduire et de diffuser l'écriture à travers le livre de colportage (20052006). Serénah Tomba (2015), quant à elle, s'intéresse à la devise individuelle et clanique pratiquée couramment dans la société punu (Gabon) en détaillant, elle aussi, leurs formes et leurs contextes d'utilisation.

\section{Problématiques transversales}

Conjointement aux analyses centrées sur un genre particulier, le travail de Jean Derive (1987) et sa présentation du fonctionnement des genres dans leur complémentarité à travers l'exemple de la littérature orale dioula de Kong (Côte d'Ivoire) est désormais un classique. Ce travail illustre la grande complexité de l'oralité et ouvre la voie à des travaux dans des perspectives transversales et transgénériques qui viennent approfondir la compréhension de la littérature orale dont on peut désormais définir la littérarité à travers des critères précis ${ }^{9}$.

En s'appuyant sur cette base, des réflexions théoriques et méthodologiques sont par la suite menées collégialement pour étayer, à partir des littératures orales africaines, la définition du statut littéraire de l'art verbal oral afin de contribuer à une vision plus globale du domaine ${ }^{10}$.

9. La thèse d'État (2015) d'Alain Sanou quant à elle est une brillante étude approfondie d'une littérature orale donnée dans une perspective ethnolinguistique et dans la suite du travail de Jean Derive (1987), en l'occurrence la littérature orale bobo (Burkina Faso). Dans la même perspective, Hélène Nelva (2004) présente la littérature orale bulgare, et César Ithier (2004), la littérature orale quechua.

10. Baumgardt \& Derive, 2008 ; Derive, 2014. 
Dans cette perspective se situe l'approfondissement de la réflexion sur la création en contexte d'oralité, sujet d'un colloque de l'International Society for the Study of Oral Literature in Africa (ISOLA) dont les actes sont publiés par Anne-Marie Dauphin et Jean Derive (2005). La question de la création est également étudiée à propos des poètes touaregs par Amalia Dragani (2012). À travers des entretiens avec plusieurs poètes touaregs, elle réunit des matériaux précieux dans ce domaine rarement étudié ${ }^{11}$. Sans que ceci ne soit forcément son objectif explicite, Amalia Dragani apporte ainsi la preuve que dans le domaine de l'oralité, on assiste souvent au mécanisme suivant : un fait est nié - simplement par ignorance, en l'occurrence l'absence d'étude approfondie et connue sur la question de la création. À partir de là et en raison de ce manque d'information, des jugements de valeur dépréciatifs sur l'oralité en général sont portés, dans le sens de l'absence de création face à la seule transmission anonyme. Cet argument est utilisé à son tour pour remettre en cause l'appartenance de la littérature orale à LA littérature, domaine défini justement par la création individuelle. On voit bien que « la boucle est bouclée ».

Par ailleurs, certaines approches apparemment centrées exclusivement sur une dimension thématique, mènent vers des ouvertures théoriques nouvelles. C'est le cas par exemple des travaux sur l'expression et les représentations de l'espace ${ }^{12}$, car ils peuvent éclairer la question de la référentialisation en littérature orale $^{13}$. Celle-ci s'établit de façon privilégiée mais non exclusive par rapport à la performance. De même, l'interrogation sur les représentations de l'altérité ${ }^{14}$ permet d'approfondir la réflexion sur les relations entre l'énonciateur et le public. En effet, la performance se réalisant dans un contexte de forte homogénéité linguistique et culturelle entre les deux pôles de la communication, l'évocation de l'Autre peut avoir lieu en son absence, ce qui n'est pas sans conséquences sur les contenus des représentations.

À travers l'ensemble des réflexions qui viennent d'être mentionnées et qui devront être complétées, on peut mieux cerner la question suivante : dans quelle mesure la situation de communication et la situation d'énonciation

11. À propos de cette population, on peut également citer la thèse dans une approche ethnolinguistique de Catherine Vaudour (2014) publiée aux Presses de l'Inalco en novembre 2018.

12. BAumgardt \& Roulon-Doko, 2010.

13. J'entends par là la façon dont un texte établit un lien spatio-temporel au réel horstexte dans lequel il s'inscrit et auquel il se réfère.

14. BAUMGARDT, 2015. 
s'inscrivent-elles dans le texte ? J'entends par « situation ou contexte de communication » la situation dans laquelle se trouvent l'énonciateur et le destinataire au moment où l'œuvre est produite. Cette situation se différencie selon la coprésence ou non des deux pôles de la communication, ce qui définit au moins partiellement la relation entre les deux et permet de distinguer la situation de communication en contexte d'oralité et en contexte d'écriture. Ainsi l'oralité, réunissant l'énonciateur et le destinataire dans un même espacetemps se distingue de ce point de vue fondamentalement de la situation de communication en contexte d'écriture, car l'écriture est par définition une médiatisation. Par ailleurs, la situation de communication en contexte d'oralité peut relever de l'oralité première ${ }^{15}$, mais également de la néo-oralité (voir infra). Par contre, la « situation d'énonciation » renvoie à une performance spécifique dans un temps/lieu précis et non reproductible à l'identique ${ }^{16}$.

Cet aperçu ne peut pas passer sous silence une autre problématique soulevée par G. Calame-Griaule dans l'ouvrage Le Renouveau du conte (1991), celle de la «néo-oralité ». Ce terme désigne, comme on le sait, l'art verbal inspiré de pratiques relevant de l'oralité première, mais produites dans des cadres nouveaux (néo-conteurs, festivals et spectacles consacrés à ce genre). Dans un contexte africain par exemple en peul (Sénégal/Mauritanie), un travail en cours, déjà mentionné, celui d'Amadou Sow, porte sur l'articulation entre l'épopée et la chanson moderne. Il promet de mieux comprendre la réactualisation de thématiques attestées traditionnellement et les liens intertextuels entre des genres très différents. Par ailleurs, la thèse de Marlène Milébou (2016), s’intéresse au répertoire d'un conteur gabonais dans des contextes de communication multiples incluant notamment le cadre de la néo-oralité. L'étude cherche à définir, à travers un corpus riche et varié, la façon dont l'énonciateur s'adapte aux différents contextes.

L'analyse du renouveau du conte, qui souligne l'importance de la néo-oralité, trouve des prolongements indirects à travers l'interrogation sur la méthode par laquelle on peut mieux documenter l'oralité. Celle-ci a des répercussions importantes, car elle ouvre la voie aux travaux sur le rôle des nouveaux médias dans la préservation de la littérature orale. On signalera ici des initiatives

15. On entend par «oralité première » la performance des œuvres dans le cadre des pratiques socio-culturelles réelles, par exemple lors de cérémonies ou rituels pratiqués dans une société donnée, sans que la performance ne soit sollicitée pour l'enregistrement, ou réalisée lors d'un spectacle.

16. Voir pour plus de détails dans BAUmgardt \& Derive, 2008, première partie. 
ponctuelles en contexte africain ou non, comme la thèse d'Agnes Kedzierska (2006), celle de Marie Lorin (2015) sur la poésie en langue peule des pêcheurs du fleuve Sénégal, la documentation filmée par Dominique Samson en Sibérie ${ }^{17}$, les travaux de Daniel Negers concernant la littérature orale télougou (Inde du Sud) ou ceux de Nicole Revel qui s'intéresse à l'épopée aux Philippines. Au-delà de ces études de cas, je voudrais mentionner les travaux spécifiquement consacrés au domaine africain de manière plus générale, comme le colloque organisé par l'ISOLA en $2012^{18}$ ou le projet « Verbum africana » animé par Daniela Merolla. Je citerai également dans ce contexte le projet en cours dans le cadre de l'ANR « Encyclopédie des Littératures en Langues africaines ${ }^{19}$ » qui porte aussi bien sur des littératures orales que sur des littératures écrites en langues africaines. L'approche ethnolinguistique sous-jacente est exprimée par l'une des questions centrales du projet, la relation entre les littératures orales et l'écriture littéraire ${ }^{20}$.

Loin d'être exhaustif, l'aperçu que je viens d'ébaucher permet d'entrevoir le réel rayonnement de l'approche ethnolinguistique en littérature orale et au-delà, à travers les nombreuses recherches réalisées dans cette perspective et dans plusieurs aires culturelles, comme en témoignent également les publications des Cahiers de littérature orale. Ces travaux intègrent d'autres approches théoriques ${ }^{21}$ et illustrent en même temps l'intérêt culturel et scientifique de l'oralité ainsi que sa richesse. S'il n'existe donc plus de doute à ce niveau et qu'on peut affirmer qu'il s'agit d'un domaine scientifique émergent, certaines difficultés persistent cependant pour le faire reconnaitre en tant que tel.

\section{Difficultés et interrogations nouvelles}

L'une des difficultés majeures, non résolue de manière satisfaisante, reste la reconnaissance de l'oralité en tant que domaine et discipline scientifiques à part entière. Ceci est dû, entre autres, au fait qu'elle se situe à un « carrefour pluridisciplinaire », entre la littérature, l'anthropologie et la linguistique.

17. On pourra se reporter également à plusieurs publications de cet auteur (notamment 2003, 2007, 2012) dans la perspective qui nous intéresse ici, l'ethnolinguistique.

$18.9^{\mathrm{e}}$ Conférence de l'ISOLA, « Oralité, nouveaux média, expérience postcoloniale », Université de Venda (Afrique du Sud), 28 juin-1 ${ }^{\text {er }}$ juillet 2012.

19. ELLAF, http://ellaf.huma-num.fr/

20. BAUMGARDT, 2017.

21. Comme le travail dans une perspective d'ethnocritique de Jean-Marie Privat (2014). 


\section{Oralité et écriture littéraire}

Cette difficulté est liée à l'articulation entre la littérature orale et une discipline proche, qui, elle, est bien reconnue sur le plan scientifique. Il est question du positionnement de la littérature orale par rapport à l'écriture littéraire. Trois tendances peuvent être distinguées.

S'il s'agit de l'écriture littéraire en alphabet arabe, on observe peu de méprises car, comme le souligne G. Calame-Griaule, la littérature orale coexiste avec la littérature écrite en ajami qui est souvent diffusée sous une forme oralisée, par exemple par des récitations mémorisées ${ }^{22}$. De ce fait, les deux modes d'expression littéraire connaissent une certaine proximité.

Les difficultés dans la perception de l'oralité par les spécialistes de l'écriture littéraire apparaissent souvent surtout du côté des littératures écrites en langues internationales. Dans cette situation et en simplifiant, on peut résumer cette perception de la manière suivante : du point de vue de l'écriture littéraire, quelque chose « manque » à la littérature orale : la création individuelle, l'auteur, l'écriture... Dans cette comparaison biaisée, la littérature orale part « perdante », alors qu'en réalité, une telle comparaison se fonde sur une méconnaissance fondamentale de l'oralité. En effet, très rares sont les chercheurs en littérature qui travaillent également sur la littérature orale ${ }^{23}$. Mais, si tel est le cas, les recherches produisent des analyses d'un grand intérêt théorique et historique, comme par exemple le travail de Frosa Bouchereau (1985) qui montre comment le conte oral en France a été une source d'inspiration de l'écriture littéraire des femmes.

Par ailleurs, toujours dans le contexte de l'écriture littéraire, un autre champ de recherche a fait émerger une interrogation nouvelle, celle sur l'écriture littéraire en langues non pas internationales, mais locales utilisant souvent l'alphabet latin ${ }^{24}$. C'est une problématique centrale du séminaire doctoral

22. Les travaux sur la littérature en ajami et plus particulièrement sur les relations entre les deux domaines sont peu documentés; à ma connaissance, il n'existe pas de bibliographie à ce sujet.

23. C'est le cas des travaux exemplaires de Jean Derive. Dans le domaine africaniste également, on peut citer des initiatives pour rapprocher les deux niveaux, par exemple RICARD, 1996 ; BAUMgardT \& UgOCHUKWU, 2005, ou encore BaUmgardT \& DerIVE, 2013, qui dirigent les actes de la journée d'étude de l'APELA organisée en 2010 au LLACAN.

24. Voir en ce qui concerne l'introduction de l'alphabet latin pour l'écriture de plusieurs langues de l'Afrique de l'Ouest, Mohamadou, 2005, p. 139 à 152. 
de l'Inalco ${ }^{25}$ «Littératures du Sud », qui s'intéresse dans une perspective transversale à la coexistence de la littérature orale avec l'écriture littéraire en langues locales. Quant à l'émergence de l'écriture littéraire contemporaine en langues africaines ${ }^{26}$, elle soulève la question de la relation de l'énonciateur à son public, relation nécessairement différente en contexte d'oralité et d'écriture littéraire.

\section{Oralité et anthropologie}

D'un autre côté, se pose le problème du positionnement de l'anthropologie par rapport à la littérature orale. À ce niveau, la question de la forme d'expression est parfois considérée comme relativement secondaire ${ }^{27}$. Cependant, une tendance nouvelle, l'anthropologie de la parole ${ }^{28}$ qui étudie les pratiques langagières en y incluant l'aspect pragmatique de la parole littéraire et qui peut donc inclure la littérature orale, promet une réelle ouverture, de même que l'ethnopoétique ${ }^{29}$ dont l'un des objectifs est de cerner l'incidence du cadre culturel sur les différentes formes d'expression de l'oralité.

\section{Oralité et linguistique}

Les difficultés concernent moins la reconnaissance de la littérature orale qui reste intéressante pour la linguistique de terrain, car les textes de littérature orale constituent des corpus de première main ; le problème se situe plutôt au niveau de la formation du chercheur qui doit être compétent dans trois disciplines à la fois, en linguistique, en anthropologie et en littérature.

La complexité engendrée par la pluridisciplinarité du domaine s'ajoute aux difficultés que je viens de mentionner et qui sont liées à la reconnaissance de la littérature orale comme une discipline, ou comme un domaine scientifique

25. Inalco et Paris 3 ; fondé par Ursula Baumgardt, Aboubakr Chraïbi, Xavier Garnier et Cécile Leguy en 2012.

26. Je citerai ici les travaux suivants, sachant que la liste n'est pas exhaustive : Bourlet, 2009 ; Chaka, 2010 ; Razaimiandrisoa, 2013 ; Garnier, 2006 ; GARNIER \& RICARD, 2006.

27. Approches littéraires de l'oralité africaine (BAUMGARDT \& UGOCHUKWU, 2005) est un titre programmatif pour équilibrer les deux perspectives.

28. Par exemple : Bornand \& Leguy, 2013.

29. L'ethnopoétique est un domaine de recherche bien ancré dans le paysage universitaire français, à travers les travaux du Groupe de Recherche en Ethnopoétique (GREP) de Paris VII et des publications comme l'ouvrage collectif $L a$ Voix actée. Pour une nouvelle ethnopoétique (2010). 
à part entière. Les répercussions sur la formation des jeunes chercheurs sont importantes, dans la mesure où ils ne trouvent pas facilement de rattachement disciplinaire, ce qui est pourtant indispensable dans les cursus universitaires et professionnels.

\section{Perspectives}

Malgré les difficultés mentionnées, l'extraordinaire richesse de la littérature orale et la fécondité de l'approche ethnolinguistique ne cessent d'être confirmées.

Pour mieux surmonter les difficultés rencontrées, plusieurs pistes sont possibles. D'un côté, une approche pluri-aréale permettra certainement d'élaborer une vision plus globale et moins fragmentée du domaine. Une telle approche est déjà pratiquée avec un grand succès depuis presque quarante ans à travers la politique éditoriale des Cabiers de littérature orale et, plus récemment, par le colloque sur l'épopée organisé par le REARE ou dans le séminaire doctoral «Littératures du Sud » que j'ai déjà mentionnés. Par ailleurs, on gagnera à renforcer des rattachements pluridisciplinaires de l'oralité par rapport à la littérature ${ }^{30}$, à la linguistique et à l'anthropologie là où cela est possible. En multipliant et en faisant converger de telles initiatives à travers des coopérations disciplinaires et aréales multiples, nous pourrons mieux consolider le champ de l'ethnolinguistique et de la littérature orale. La création de formations pluridisciplinaires va dans ce sens. Ainsi, on peut espérer par exemple que le Master «Oralité et Anthropologie », ouvert à l'Inalco en coopération avec Paris 7 en 2014/2015 soit un premier pas vers un ancrage progressif des formations en littérature orale dans le paysage universitaire français. De même le programme de recherche « Encyclopédie des Littératures en Langues africaines » (ELLAF) qui intègre la littérature orale, la littérature écrite en alphabet latin ${ }^{31}$ et en ajami apportera une plus grande visibilité au domaine et une légitimité scientifique accrue.

30. Ainsi, l'Association pour l'Étude des Littératures africaines (APELA) intègre régulièrement des travaux sur la littérature orale dans ses programmes.

31 . Voir note 24. 


\section{Sources et références citées}

BA Alpha Oumarou, 2011, L'épopée peule du Fouladou (Sénégal) : texte et contexte, Thèse de doctorat sous la direction de Baumgardt Ursula, Institut national des langues et civilisations orientales, Paris, $527 \mathrm{p}$.

BARry Alpha Ousmane, 2011, L'épopée peule du Funta Jalo : de l'éloge à l'amplification rhétorique, Karthala, Paris, 359 p.

BAUmgardt Ursula, 2000, Une conteuse peule et son répertoire: Goggo Addi de Garoua, Cameroun: Textes et analyses, Karthala, Paris, 548 p.

BAUMgARDT Ursula, 2010, « La littérature orale n'est pas un vase clos » in BAUmgardt Ursula \& Derive Jean (dir.), Littératures orales africaines : perspectives théoriques et méthodologiques, Karthala, Paris, p. 245-270.

BAUMGARDT Ursula (dir.), 2014, Représentations de l'altérité dans la littérature orale africaine, Karthala, Paris, 309 p.

BAUmgardt Ursula (dir.), 2017, Littératures en langues africaines. Production et diffusion, Karthala, Paris, 361 p.

Baumgardt Ursula \& Derive Jean (dir.), 2005, Paroles nomades : écrits d'ethnolinguistique africaine. En hommage à Christiane Seydou, Karthala, Paris, 538 p.

Baumgardt Ursula \& Derive Jean (dir.), 2008, Littératures orales africaines : perspectives théoriques et méthodologiques, Karthala, Paris, 439 p.

Baumgardt Ursula \& Derive Jean (dir.), 2013, Littérature africaine et oralité, Karthala, Paris, $164 \mathrm{p}$.

Baumgardt Ursula \& Roulon-Doko Paulette (dir.), 2010, L'expression de l'espace dans les langues africaines, tome 2, Société des africanistes (coll. Journal des Africanistes), Paris, $443 \mathrm{p}$.

Baumgardt Ursula \& Ugochukwu Françoise (dir.), 2005, Approches littéraires de l'oralité africaine : en hommage à Jean Derive, Karthala, Paris, $334 \mathrm{p}$. 
Bornand Sandra, 2005, Le discours du griot généalogiste chez les Zarma du Niger, Karthala, Paris, 457 p.

Bornand Sandra \& Leguy Cécile, 2013, Anthropologie des pratiques langagières, Armand Colin (coll. Collection U. Sciences humaines \& sociales), Paris, 205 p.

Bouchereau Frosa, 1985, Généalogie du conte ou le conte dans son expression orale et écrite, Université de Rouen, Mémoire de maîtrise, Rouen.

Bourlet Mélanie, 2009, L'émergence d'une littérature écrite dans une langue africaine : l'exemple du poulâr (sénégal/Mauritanie), Thèse de doctorat sous la direction de Baumgardt Ursula, Institut national des langues et civilisations orientales, Paris.

Calame Claude, Dupont Florence, Lortat-Jacob Bernard \& Manca Maria (dir.), 2010, La voix actée : pour une nouvelle ethnopoétique, Éditions Kimé, Paris, 327 p.

CAlAme-Griaule Geneviève, 1969, Le thème de l'arbre dans les contes africains, 3 tomes, SELAF, Paris, $92+137+208$ p.

Calame-Griaule Geneviève, 1970, « Pour une étude ethnolinguistique des littératures orales africaines » in Pottier Bernard (dir.), Langages, $\mathrm{n}^{\circ} 18$, p. 22-47, DOI : $10.3406 /$ lgge.1970.2026.

Calame-Griaule Geneviève, 1987, Des Cauris au marché : essais sur des contes africains, Société des africanistes, Paris, 293 p.

Calame-Griaule Geneviève (dir.), 1991, Le Renouveau du conte, Éditions du CNRS, Paris, 449 p.

Calame-Griaule Geneviève, 2006, Contes dogon du Mali, Karthala/Inalco (coll. Paroles en miroir), Paris, 243 p.

Chaka Limakatso Elizabeth, 2010, Pitseng de Thomas Mofolo, roman sesotho (Afrique australe) : texte et contexte, Thèse de doctorat sous la direction de Baumgardt Ursula, Institut national des langues et civilisations orientales, Paris, $330+268$ p. 
Collectif Langage et culture en Afrique de l'ouest (dir.), 1989, Graines de parole : puissance du verbe et traditions orales : textes offerts à Geneviève Calame-Griaule, CNRS Éditions, Paris, France, 441 p.

Dauphin-Tinturier Anne-Marie \& Derive Jean (dir.), 2005, Oralité africaine et création: actes du colloque de l'ISOLA, 10-12 juillet 2002, Karthala, Paris, $348 \mathrm{p}$.

DerIVE Jean, 1986, Le fonctionnement sociologique de la littérature orale : l'exemple des Dioula de Kong (Côte d'Ivoire), Thèse de doctorat sous la direction de Calame-Griaule Geneviève, Paris, 2326 p.

Derive Jean (dir.), 2002, L'épopée : unité et diversité d'un genre, Karthala, Paris, $262 \mathrm{p}$.

Derive Jean (dir.), 2012, Chanter l'amour en pays dioula : badinage, sexe et jalousie, Classiques africains, Paris, $247 \mathrm{p}$.

Derive Jean, 2014, L'art du verbe dans l'oralité africaine, L'Harmattan, Paris, $224 \mathrm{p}$.

Dieng Bassirou \& Kesteloot Lilyan, 1997, Les Épopées d'Afrique noire, Karthala/Unesco, Paris, 626 p.

Dragani Amalia, 2012, Interno tuareg. Etnografia partecipativa dei poetit nomadi del Niger, Arcane, Roma, 160 p.

Dumestre Gérard (dir.), 1979, La Geste de Ségou racontée par des Griots Bambara, Armand Colin, Paris, 417 p.

Durand-Dastès Vincent, 2014, « La Grande Muraille des contes : une collecte géante de littérature populaire en chine à la fin $\mathrm{du} \mathrm{xx}^{\mathrm{e}}$ siècle et sa publication », Carreau de la BULAC, https://bulac.hypotheses.org/1676.

ELLAF, s. d., Encyclopédie des littératures et langues africaines, http://ellaf.humanum.fr/.

FAYE Amade, 1997, Le thème de la mort dans la littérature Seereer : essai, Nouvelles éditions africaines du Sénégal, Dakar, 311 p. 
GARNIER Xavier, 2006, Le roman swahili : la notion de «littérature mineure » à l'épreuve, Karthala, Paris, 242 p.

GARnier Xavier \& RicARd Alain (dir.), 2006, L'effet roman : arrivée du roman dans les langues d'Afrique, L'Harmattan, Paris, $311 \mathrm{p}$.

GÖRÖG-KARADY Veronika (dir.), 1994, Le mariage dans les contes africains : études et anthologie, Karthala, Paris, 225 p.

GÖRÖG-KARADY Veronika, 1997, L'univers familial dans les contes africains : liens de sang, liens d'alliance, L'Harmattan, Paris, 284 p.

Görög-Karady Veronika \& BAUMgardt Ursula (dir.), 1988, L'enfant dans les contes africains, Conseil international de la langue française, Paris, 189 p.

Görög-Karady Veronika, Bouillet Catherine \& TAmari Tal, 1992, Bibliographie annotée littérature orale d'Afrique noire, Conseil international de la langue française, Paris, $367 \mathrm{p}$.

Görög-Karady Veronika, Karady Victor \& Chiche Michèle, 1981, Littérature orale d'Afrique noire : bibliographie analytique, G.-P. Maisonneuve et Larose, Paris, France, 394 p.

Görög-Karady Veronika, Platiel Suzanne, Rey-Hulman Diana, Seydou Christiane \& Calame-Griaule Geneviève, 1980, Histoires d'enfants terribles : Afrique noire, études et anthologie, G.-P. Maisonneuve et Larose, Paris, 301 p.

GÖRÖG-Karady Veronika \& SEYDou Christiane (dir.), 2001, La fille difficile : un conte-type africain, Éditions du CNRS, Paris, 492 p.

Itier César, 2004, La littérature orale quechua de la région de Cuzco, Pérou, Karthala/Inalco, Paris, 238 p.

Jansen Jan, 2001, Epopée, histoire, société : le cas de Soundjata : Mali et Guinée, Karthala (coll. Collection Hommes et sociétés), Paris, 307 p.

Kedzierska-Manzon Agnès, 2014, Chasseurs mandingues : violence, pouvoir et religion en Afrique de l'Ouest, Karthala, Paris, 256 p. 
Keita Abdoulaye, 2010, La poésie orale d'exhortation : l'exemple des bàkkì des lutteurs wolof (Sénégal), Atelier national de reproduction des thèses, Lille.

Keita Abdoulaye, 2010, « Le bàkku ou l'épique dans l'arène de la lutte en milieu wolof » in Annales de la faculté des lettres et sciences humaines, vol. 40, p. 211-229.

Kesteloot Lilyan (dir.), 1972, Da Monzon de Segou : épopée bambara, 4 tomes, réédité en 1993 chez l'Harmattan en 2 volumes, Nathan, Paris, $63+63+79+63 \mathrm{p}$.

Kesteloot Lilyan \& Dumestre Gérard (dir.), 1975, La Prise de Dionkoloni : épisode de l'épopée bambara, Armand Colin, Paris, 183 p.

Leguy Cécile, 2001, Le proverbe chez les Bwa du Mali : parole africaine en situation d'énonciation, Karthala, Paris, 323 p.

Lorin Marie, 2015, La poésie orale peule des pêcheurs de la vallée de fleuve Sénégal (Pekaan) : approche géopoétique, Thèse de doctorat sous la diretion de Baumgardt Ursula, Institut national des langues et civilisations orientales, Paris.

Merolla daniela, s. d., Verba Africana, African Languages and Oral Literatures: Video Documentation and Digital Materials, http://verbafricana.org/.

Milebou Ndjave Kelly Marlène, 2016, Performances de Brice Senah Ambenga, un conteur orungu du Gabon en contextes multiples, Thèse de doctorat sous la direction de Baumgardt Ursula, Institut national des langues et civilisations orientales, Paris.

Mohamadou Aliou, 2005, « Si Bamako m'était conté... À propos de la transcription de l'orthographe du peul » in BAumgardT Ursula \& Derive Jean (dir.), Paroles nomades : écrits d'ethnolinguistique africaine : En hommage à Christiane Seydou, Karthala, Paris, p. 139-152.

Ndiaye Oumar, 2010, La notion de répertoire dans l'oeuvre de Guéllâye, poète épique des pếcheurs haalpulaar du Foûta-Tôro (Mauritanie/Sénégal), Thèse de doctorat sous la direction de Baumgardt Ursula, Institut national des langues et civilisations orientales, Paris. 
Negers Daniel, 1995, « La dimension politique dans l'émergence d'une forme narrative populaire à l'époque moderne : le Burrakatha d'Andhra Pradesh », in Collectif, Traditions orales dans le Monde Indien, Édition de l'EHESS, Paris, p. 103-125.

Negers Daniel, 1997, Le Burrakatha d'Andhra Pradesh (Inde). Essai de description d'une forme narrative théatralisée en langue télougoue. L'importance de l'expression littéraire dans la communication et la culture populaires, sans mention de l'éditeur et du lieu, $810 \mathrm{p}$.

Negers Daniel, 2004, « De l'expression orale au genre littéraire, L'exemple du burrakatha d'Andhra Pradesh » in Montaut Annie (dir.), Littérature et poétiques pluriculturelles en Asie du Sud, Édition de l'EHESS, Paris, p. 157-179.

Nelva-Courtin Hélène (dir.), 2007, La littérature orale bulgare, Karthala/ Inalco, Paris, $276 \mathrm{p}$.

Nkejabahizi J.-C., 2013, Les wellérismes du Rwanda. Approche ethnolinguistique, Éditions de l'université nationale du Rwanda, Butare, $303 \mathrm{p}$.

Platiel Suzy, 1984, La Fille volage et autres contes du pays san, Armand Colin (coll. Africains), Paris, $342 \mathrm{p}$.

Privat Jean-Marie, 2014, « La lettre et le panier. Ethnocritique de Flaubert » in Ethnologie francaise, ${ }^{\circ} 4$, vol. 44, p. 651-661.

Razaimiandrisoa Nirina, 2013, Représentations de la société malgache des années 30 : Alfred Ramandiamanana (1886-1939), Thèse de doctorat sous la direction de Baumgardt Ursula \& Rajaonarimanana N., Institut national des langues et civilisations orientales, Paris.

Revel Nicole (dir.), 1990-1992, Fleurs de paroles : histoire naturelle palawan, 3 tomes, Peeters/SELAF, Paris, $374+322+208$ p.

Revel Nicole, 2013, "The Philippine Epic and Ballads Multimedia Archive" in Oral Tradition, $\mathrm{n}^{\circ}$ 2, vol. 28, pp. 371-378. 
Revel Nicole, Railling Patricia Ann \& Senné Nathalie (dir.), 2000, La quête en épouse : Mämiminbin, une épopée palawan chantée par Mäsinu [The Quest for a Wife : Mämiminbin, a Palawan Epic Sung by Mäsinu], édition palawan-français-anglais, UNESCO/L'Asiathèque, Paris, $428 \mathrm{p}$.

RICARD Alain, 1995, Littératures d'Afrique noire: des langues aux livres, Karthala, Paris, 304 p.

Roulon-Doko Paulette (dir.), 1977, Wanto... et l'origine des choses : contes d'origine... et autres contes Gbaya-Kara : Centrafrique, Edicef: CILF, Paris, $143 \mathrm{p}$.

Ruelland Suzanne, 1973, La fille sans mains. Analyse de dix-neuf versions africaines du conte de type 706, SELAF, Paris, $209 \mathrm{p}$.

Samson Normand De Chambourg Dominique, 2003, « Du frère tombé du ciel au frère retrouvé : brèves apostilles à la littérature mansie » in Études finno-ougriennes, vol. 34, p. 69-106.

Samson Normand De Chambourg Dominique, 2007, « De la chance à l'identité khantye : les jeux de l'Ours » in GLowczewski Barbara \& Henry Rosita (dir.), Le défi indigène : entre spectacle et politique, Aux lieux d'être, Montreuil, p. 279-299.

Samson Normand De Chambourg Dominique, 2012, «L'Ours, la lune et les pentecôtistes. Du paysage religieux des Khantys septentrionaux au début du $\mathrm{XXI}^{\mathrm{e}}$ siècle » in Géry Catherine \& Samson Normand De Снambourg Dominique (dir.), Festchrift en l'honneur d'Anne-Victoire Charrin, Publication Langues'O, Paris, p. 69-109.

Sanou Alain, 2015, La notion de parole chez les Bobos, Thèse d'état, Université de Ouagadougou.

Servan-Schreiber Catherine, 1981, « Le dormeur et le joli-coeur : tradition de plaisanteries dans les chants bhojpuri (Inde du Nord) » in Cabiers de littérature orale, $\mathrm{n}^{\circ} 6$, p. 17-40.

Servan-Schreiber Catherine, 1984, « Le livret de colportage en Inde du Nord » in Revue française d'histoire du livre, vol. 126-127, p. 251-256. 
52 Geneviève Calame-Griaule $-n^{\circ} 83$

Servan-Schreiber Catherine, 1999, Chanteurs itinérants en Inde du Nord: la tradition orale bhojpuri, L'Harmattan, Paris, 415 p.

Seydou Christiane (dir.), 1972, Silâmaka Poullôri : récit épique peul, Armand Colin, Paris, 277 p.

Seydou Christiane (dir.), 1976, La Geste de Ham-Bodêdio ou Hama le Rouge, Armand Colin, Paris, 419 p.

SEydou Christiane (dir.), 2010, Profils de femmes dans les récits épiques peuls : Mali-Niger, Karthala, Paris, 273 p.

Seydou Christiane (dir.), 2011, L'épopée peule de Boûbou Ardo Galo, héros et rebelle, Karthala/Inalco, Paris, 278 p.

Tомва Diogo Amevi Christine Cerena, 2015, Étude d'un genre de la littérature orale : la devise (kûmbì) chez les Punu du Gabon, Thèse de doctorat sous la direction de Baumgardt Ursula \& Racine Odile, Institut national des langues et civilisations orientales, Paris.

Toulouze Eva, 2003, "Forest Nenets as a Dual Language Minority, Multiethnic Communities in the Past and Present" in Pro Ethnologia, vol. 15, p. 95-108.

Ugochukwu Françoise (dir.), 1992, Contes igbo du Nigeria : de la brousse à la rivière, Karthala, Paris, $351 \mathrm{p}$.

Ugochukwu Françoise, 2006, Contes igbo de la Tortue : Nigéria, Karthala, Paris, $123 \mathrm{p}$.

VAudour Catherine, 2014, L'évolution des pratiques matrimoniales chez les Ker-Ajjer (Sud-est de l'Algérie), étude ethnolinguistique d'une alchimie entre tradition et modernité, Institut national des langues et civilisations orientales, Thèse de doctorat publiée en 2018, Les Cérémonies du mariage chez les KelAjjer du Sud-Est de l'Algérie, étude ethnolinguistique d'une alchimie entre tradition et modernité, Presses de l'Inalco, Paris. 
Résumé : cet article montre que l'approche ethnolinguistique de la littérature orale initiée par Geneviève Calame-Griaule est très féconde et qu'elle trouve des applications dans des dimensions pluriaréales. Elle est utilisée pour l'analyse de tous les genres de la littérature orale. Cette vitalité des travaux prouve en même temps l'importance de la littérature orale elle-même. Cependant, malgré cette vitalité, plusieurs problèmes persistent. En effet, l'une des difficultés majeures non résolue de manière satisfaisante reste la reconnaissance de l'oralité en tant que domaine et discipline scientifiques à part entière. Or, cette reconnaissance est indispensable pour permettre aux jeunes chercheurs de trouver des rattachements disciplinaires dans les cursus universitaires et professionnels. Plusieurs pistes pour résoudre ce problème sont proposées. D’un côté, une approche pluri-aréale permet certainement d'élaborer une vision plus globale et moins fragmentée du domaine, approche qui est déjà pratiquée avec un grand succès depuis presque quarante ans à travers la politique éditoriale des Cabiers de littérature orale. Par ailleurs, on peut chercher à renforcer des rattachements pluridisciplinaires de l'oralité par rapport à la littérature, à la linguistique et à l'anthropologie là où c'est possible.

Mots-clefs : ethnolinguistique, oralité, littérature orale, approche pluri-aréale et pluridisciplinaire.

\section{Acknowledging of Oral Literature as a Full-Fledged Scientific Domain and Discipline, Pluriareal Approach}

Abstract: this article shows that the ethnolinguistic approach of oral literature initiated by Genevieve Calame-Griaule has been very fruitful: it has already been applied in pluriareal dimensions and is now used in the analysis of all oral literary genres, proving the importance of oral literature. This vitality has not eliminated all the problems. One of the major unresolved issues is that of the acknowledging of oral literature as a fullfledged scientific domain and discipline. Yet, acknowledging it as a separate discipline is crucial if one wants to allow young researchers to find the right affliations in university curricula and professional career. Here are some suggested directions to resolve this problem. First, a pluriareal approach would certainly help to produce a more global and less fragmented vision of the domain, as proved by its highly successful application in the editorial policy of the Cahiers de littérature orale for more than twenty years. Moreover, one could 
CAHIERS DE LITTÉRATURE ORALE

54 Geneviève Calame-Griaule - $n^{\circ} 83$

seek to reinforce already existing links between orality and literature, linguistics and anthropology, wherever possible.

Keywords: ethnolinguistics, orality, oral literature, pluriareal approach.

\section{Note sur l'auteur}

Ursula Baumgardt est professeur à l'Inalco de «Oralité et littérature africaine », depuis janvier 2017, membre de l'équipe de recherche PLIDAM, Inalco/Paris Sorbonne Cité et participe au groupe de réflexion sur les aires culturelles et à la commission des transversaux. Elle est responsable avec Frosa Bouchereau du Master Oralité et a coordonné le projet ANR « Encyclopédie des Littératures en Langues africaines » (ELLAF) de janvier 2014 à décembre 2017. 